\title{
ON THE CALKIN ALGEBRA AND THE COVERING HOMOTOPY PROPERTY
}

\author{
BY
}

JOHN B. CONWAY(1)

\begin{abstract}
Let $\mathcal{H}$ be a separable Hilbert space, $\mathscr{B}(\mathcal{H})$ the bounded operators on $\mathcal{H}, \mathcal{K}$ the ideal of compact operators, and $\pi$ the natural map from $B(H)$ onto the Calkin algebra $\mathscr{B}(\mathcal{H}) / \mathcal{K}$. Suppose $X$ is a compact metric space and $\Phi: C(X) \times[0,1] \rightarrow B(H) / K$ is a continuous function such that $\Phi(\cdot, t)$ is a $*$-isomorphism for each $t$ and such that there is a *-isomorphism $\psi: C(X) \rightarrow \$(H)$ with $\pi \psi(\cdot)=\Phi(\cdot, 0)$. It is shown in this paper that if $X$ is a simple Jordan curve, a simple closed Jordan curve, or a totally disconnected metric space then there is a continuous map $\Psi: C(X) \times[0,1] \rightarrow \mathscr{B}(\mathcal{H})$ such that $\pi \Psi=\Phi$ and $\boldsymbol{\Psi}(\cdot, 0)=\psi(\cdot)$. Furthermore if $X$ is the disjoint union of two spaces that both have this property, then $X$ itself has this property.
\end{abstract}

If $\mathcal{H}$ is a separable Hilbert space, $\mathcal{K}$ is the set of compact operators on $\mathcal{H}$, and $\pi$ is the natural map from $B(H)$ onto the Calkin algebra $\mathfrak{B}(\mathcal{H}) / \mathcal{K}$ then a compact metric space $X$ is said to have the $C^{*}$-covering homotopy property whenever the following holds: for any continuous map $\Phi: C(X) \times[0,1] \rightarrow$ $\mathfrak{B}(\mathcal{H}), / \mathcal{K}$ such that $\Phi(\cdot, t)$ is a *-isomorphism for each $t$ and there is a *-isomorphism $\psi: C(X) \rightarrow \mathfrak{B}(\mathcal{H})$ with $\pi \psi(\cdot)=\Phi(\cdot, 0)$ then there is a continuous map $\Psi: C(X) \times[0,1] \rightarrow \mathscr{B}(\mathcal{H})$ such that $\Psi(\cdot, t)$ is a *-isomorphism for each t, $\pi \Psi=\Phi$, and $\psi(\cdot)=\Psi(\cdot, 0)$. In this paper it is shown that $X$ has the $C^{*}$ covering homotopy property when $X$ is an interval, a circle, a totally disconnected space, or the disjoint union or two spaces which have the property.

Here $C(X)$ is the $C^{*}$-algebra of all continuous complex valued functions on $X$, and an isomorphism is understood to take the identity of the domain onto the identity of the range.

The motivation to study this problem stems from the recent papers of Brown, Douglas and Fillmore [3], [4]. Indeed, if it can be shown that every compact metric space has the $C^{*}$-covering homotopy property, then this will

Received by the editors July 8, 1974 .

AMS (MOS) subject classifications (1970). Primary 46L05; Secondary 55D10.

Key words and phrases. Operators on a Hilbert space, Calkin algebra, covering homotopy property.

(1) The author acknowledges the partial support of the National Science Foundation under Grant No. 28601 during the preparation of this paper. 
give a direct proof that their group $\operatorname{Ext}(X)$ is a homotopy invariant and will shed more light on its nature [6]. Moreover the fact that a two point set and the circle have the $C^{*}$-covering homotopy property has been used to explore the relationship between $\operatorname{Ext}(X)$ and algebraic $K$-theory [2].

Before going further it seems appropriate to say a few words concerning terminology. The standard practice is to discuss maps, not compact metric spaces, which have the covering homotopy property. It would be better to say that $\pi$ has the $C^{*}$-covering homotopy property if the above definition is satisfied not only for the algebras $C(X)$, but for arbitrary $C^{*}$-algebras. Then one could define $\pi$ to have this property relative to an algebra $C(X)$, and it is proved here that this is the case when $X$ is an interval, a circle, or a totally disconnected space. Nevertheless, this rational nomenclature will not be adopted here in order to achieve a certain economy in terminology.

It is easy to see that the $C^{*}$-covering homotopy property is a homeomorphic invariant. So the results presented here imply that simple and closed simple curves have the $C^{*}$-covering homotopy property. Beyond these examples, totally disconnected spaces, and finite disjoint unions of these, there are no further spaces known to have this property. Indeed, it is not known whether a fiqure eight, a circle with a spoke, or the set $[-1,0] \cup\left\{2^{-n}: n \geq 1\right\}$ has the $C^{*}$-covering homotopy property.

The proof of the first lemma is straightforward.

1. Lemma. A compact subset $X$ of the complex plane has the $C^{*}$-covering homotopy property if and only if for any curve $\{a(t)\}$ of normal elements in $B(H) / K$ with o $(a(t))=X$ for all $t$ and such that there is a normal operator $A_{0}$ in $B(H)$ with $\sigma\left(A_{0}\right)=X$ and $\pi\left(A_{0}\right)=a(0)$, then there is a curve $\{A(t)\}$ of normal operators in $B(H)$ such that $\sigma(A(t))=X$ for all $t, A(0)=A_{0}$, and $\pi(A(t))=a(t)$.

2. Theorem. If $X$ is an interval then $X$ has the $C^{*}$-covering homotopy property.

Proof. Suppose $X=[0,1]$ and let $r$ be the retraction of $\mathbf{R}$ onto $[0,1]$. According to a theorem of Bartle and Graves [7], if $X$ and $\mathcal{Y}$ are Banach spaces and $T: X \rightarrow Y$ is a continuous linear map of $\mathcal{X}$ onto $Y$ and $T\left(x_{0}\right)=$ $y_{0}$ for some point $x_{0}$ in $\mathcal{X}$, then there is a continuous map $\phi: \mathcal{Y} \rightarrow X$ such that $T(\phi(y))=y$ for all $y$ in $Y$ and $\phi\left(y_{0}\right)=x_{0}$. If $X$ and $Y$ are the real Banach spaces consisting of the selfadjoint elements in $B(\mathcal{H})$ and $B(H) / K$ and $T=\pi$, then the hypotheses of the Bartle-Graves theorem are satisified. So if $\{a(t)\}$ is a path of selfadjoint elements in $B(H) / K$ with $o(a(t))=[0,1]$ for 
each $t$ and if $A_{0}$ is a selfadjoint operator in $B(H)$ with $\sigma\left(A_{0}\right)=[0,1]$ and $\pi\left(A_{0}\right)=a(0)$, then the Bartle-Graves theorem implies that there is a continuous path $\{A(t)\}$ of selfadjoint operators such that $\pi(A(t))=a(t)$ and $A(0)=$ $A_{0}$. If $B(t)=r(A(t))$ then $B(t)$ is selfadjoint, $B(0)=A_{0}$, and $\sigma(B(t))=$ $r[\sigma(A(t))]=[0,1]$. (This last equality holds because $\left.[0,1]=\sigma(a(t)) \subset \sigma(A(t))_{0}\right)$

For $t$ fixed put $A=A(t), B=B(t)$. By Weyl's theorem [8, p. 367], $\sigma(A) \backslash[0,1]$ consists of isolated eigenvalues of finite multiplicity. So the spectral theorem gives that

$$
A=H+\sum_{n=1}^{\infty} \lambda_{n} E_{n}+\sum_{n=1}^{\infty} \mu_{n} F_{n}
$$

where $\left\{E_{1}, F_{1}, E_{2}, F_{2}, \ldots\right\}$ are mutually orthogonal projections of finite rank, $\left\{\lambda_{n}\right\}$ is a sequence decreasing to $1,\left\{\mu_{n}\right\}$ is a sequence increasing to 0 , and $H$ is a selfadjoint operator with $\sigma(H)=[0,1]$ and $\operatorname{ker} H=(E+F)(\mathcal{H})$ where $E=\Sigma E_{n}, F=\Sigma F_{n}$. Since $B=r(A)=H+E$,

$$
B-A=\sum_{n=1}^{\infty}\left(1-\lambda_{n}\right) E_{n}-\sum_{n=1}^{\infty} \mu_{n} F_{n}
$$

and each of these sums is a compact operator; therefore $\pi(A)=\pi(B)$.

Finally, $\|A(\cdot)\|$ is a continuous function on $[0,1]$ so there is an interval $[a, b]$ such that $\sigma(A(t)) \subset[a, b]$ for each $t$. Since $\{p(A(t))\}$ is a continuous path for any polynomial $p$ and $r$ can be uniformly approximated by polynomials on $[a, b],\{r(B(t))\}$ is a continuous path and the proof is complete by virtue of Lemma 1.

3. Theorem. A circle has the $C^{*}$-covering homotopy property.

Proof. Suppose $X$ is the unit circle and that $\{u(t)\}$ is a path of unitary elements in $B(\mathcal{H}) / \mathcal{K}$ such that $\sigma(u(t))=X$ for all $t$ and there is a unitary operator $U_{0}$ in $B(H)$ with $\pi\left(U_{0}\right)=u(0)$. By the Bartle-Graves theorem there is a path $\{A(t)\}$ in $B(\mathcal{H})$ such that $\pi(A(t))=u(t)$ and $A(0)=U_{0}$. (Notice that it is not necessarily true that $A(t)$ is normal.) Let $A(t)=W(t) B(t)$ be the polar decomposition of $A(t)$ and choose $\delta>0$ such that $\|A(s)-A(t)\|<$ 1 whenever $|s-t|<\delta$. If $0 \leq t \leq \delta$ then $\left\|U_{0}-A(t)\right\|<1$ and so $A(t)$ is invertible; hence $B(t)=\left[A(t)^{*} A(t)\right]^{1 / 2}$ is invertible and $W(t)$ is a unitary. Furthermore, $\left\{B(t)^{-1}\right\}$ is continuous so $\{W(t)\}$ is continuous on $[0, \delta]$. Since $\pi(B(t))^{2}=\pi\left(A(t)^{*}\right)_{\pi}(A(t))=u(t)^{*} u(t)=1, \pi(W(t))=u(t)$ for $0 \leq t \leq \delta$. Let $U(t)=W(t)$ for $0 \leq t \leq \delta$. Then $U(0)=W(0)=A(0)=U_{0}$. Also, $\pi(U(\delta))=$ A $\pi(A(\delta))$ implies that $U(\delta)-A(\delta)=K$ is compact. 
For $\delta \leq t \leq 2 \delta$,

$$
\|[A(t)+K]-U(\delta)\|<1
$$

so that $A(t)+K$ is invertible. Applying the same technique as above extends $\{U(t)\}$ continuously to $[0,2 \delta]$. An induction argument and Lemma 1 complete the proof.

It is known that the spectral radius is an upper semicontinuous function on $B(H)$ and it is continuous on the normal elements (because it equals the norm). This is essentially the content of the next lemma although the particular phrasing is needed for certain estimates which are to be made below. (See [5, Problem 86].)

4. Lemma. If $A$ and $B \in \mathfrak{B}(\mathcal{H})$ and $A$ is normal then

$$
\sigma(B) \subset\{\lambda: \operatorname{dist}(\lambda, \sigma(A)) \leq\|A-B\|\} \text {. }
$$

Proof. The proof uses the fact that if $x$ and $y$ are elements of a Banach algebra with identity, $x$ is invertible and $\|x-y\|<\left\|x^{-1}\right\|^{-1}$ then $y$ is also invertible. If $\|A-B\|<\operatorname{dist}(\lambda, \sigma(A)) \Rightarrow \inf \{|z-\lambda|: z \in \sigma(A)\}$ then $\|A-B\|^{-1}>\sup \left\{|z-\lambda|^{-1}: z \in \sigma(A)\right\}=\left\|(\lambda-A)^{-1}\right\|$ since $A$ is normal. Using the above fact with $x=(\lambda-A)$ and $y=(\lambda-B)$, it follows that $\lambda \notin \sigma(B)$. This proves the lemma.

If in the next lemma the part of the hypothesis and the conclusion which involves the path $\{Q(t)\}$ is deleted then this lemma states that a two point space has the $C^{*}$-covering homotopy property. The role of $\{Q(t)\}$ is to make it possible to prove that any. finite set and, eventually, all totally disconnected spaces have the $C^{*}$-covering homotopy property.

5. Lemma. Let $\{Q(t)\}$ be a path of projections in $B(H)$ and $\{p(t)\}$ a path of projections in $B(H) / K$ such that $\pi(Q(t)) \geq p(t)$ for all t. If $P_{0}$ is a pro. jection in $B(H)$ with $P_{0} \leq Q(0)$ and $\pi\left(P_{0}\right)=p(0)$ then there is a path of projections $\{P(t)\}$ in $B(H)$ with:

(a) $P(0)=P_{0}$,

(b) $\pi(P(t))=p(t)$,

(c) $P(t) \leq Q(t)$ for all $t$.

Proof. Again applying the Bartle-Graves theorem, there is a path $\{A(t)\}$ of selfadjoint operators in $B(\mathcal{H})$ such that $A(0)=P_{0}$ and $\pi(A(t))=p(t)$. If $B(t)=Q(t) A(t) Q(t)$ then $\{B(t)\}$ is a path of selfadjoint operators, $\pi(B(t))=$ $p(t), B(0)=P_{0}$, and $Q(t) B(t)=B(t) Q(t)=B(t)$ for all $t$. Choose a $\delta>0$ such that $\|B(s)-B(t)\|<1 / 2$ whenever $|s-t|<\delta$. It follows from the preceding lemma that 


$$
\sigma(B(t)) \subset\left(-\frac{1}{2}, \frac{1}{2}\right) \cup\left(\frac{1}{2}, \frac{3}{2}\right)
$$

for $0 \leq t \leq \delta$ since $B(0)=P_{0}$ is a projection. Hence $\chi$, the characteristic function of $(1 / 2,3 / 2)$, is continuous on the spectrum of $B(t)$ for $0 \leq t \leq \delta$. Put $P(t)=\chi(B(t))$ for $0 \leq t \leq \delta$. It follows that $P(t)$ is a projection and $P(0)=P_{0}$. If $0 \leq t_{0} \leq \delta$ then the fact that $\chi$ can be uniformly approximated by polynomials on $\sigma\left(B\left(t_{0}\right)\right)$ implies that $\{P(t)\}$ is continuous at $t_{0}$. Also, if $f$ is a polynomial that vanishes at zero then $Q(t) f(B(t))=f(B(t)) Q(t)=f(B(t))$. Since $\chi$ can be approximated by such polynomials, $P(t) \leq Q(t)$ for $0 \leq t \leq \delta$. This same argument, combined with the fact that $\chi(p(t))=p(t)$ gives that $\pi(P(t))=p(t)$.

In particular, since $\pi(P(\delta))=\pi(B(\delta))$ it follows that $K=B(\delta)-P(\delta) \epsilon$ K. Also for $\delta \leq t \leq 2 \delta$,

$$
\|[B(t)-K]-P(\delta)\| \leq 1 / 2,
$$

so that

$$
\sigma(B(t)-K) \subset\left(-\frac{1}{2}, \frac{1}{2}\right) \cup\left(\frac{1}{2}, \frac{3}{2}\right)
$$

Therefore $P(t)=\chi(B(t)-K)$ for $\delta \leq t \leq 2 \delta$ defines a continuous path on $[\delta, 2 \delta]$ which agrees with the previous definition of $P(\delta)$ when $t=\delta$. As above, it is easy to see that $\{P(t)\}$ has the required properties. An induction argument completes the proof.

6. Theorem. If $X$ is totally disconnected then $X$ hais the $C^{*}$-covering homotopy property.

Proof. Suppose (for notational convenience) that the diameter of $X$ is one. Then there are subsets $\left\{S_{n k}: 1 \leq k \leq 2^{n}, n \geq 1\right\}$ of $X$ which are simultaneously open and closed and such that for $n=1,2, \ldots$ :

(a) $\bigcup_{k=1}^{2^{n}} s_{n k}=X$;

(b) $S_{n k}=S_{n+1,2 k-1} \cup S_{n+1,2 k}$;

(c) $S_{n k} \cap S_{n j}=\square$ if $j \neq k$;

(d) diameter $\left(S_{n k}\right) \leq(2 / 3)^{n}$.

If $\chi_{n k}=$ the characteristic function of $S_{n k}$ then $\chi_{n k} \in C(X)$.

Let $\Phi: C(X) \times[0,1] \rightarrow B(\mathcal{H}) / \mathcal{K}$ be a continuous map such that $\Phi(\cdot, t)$ is a *-isomorphism for each $t$, and let $\psi: C(X) \rightarrow \Re(\mathcal{H})$ be a *-isomorphism such that $\pi \psi(\cdot)=\Phi(\cdot, 0)$. Using Lemma 5 and mathematical induction it can be shown that there are paths of projections $\left\{P_{n k}(t)\right\}$ in $B(\mathcal{H})$ with $P_{n k}(0)=$ $\psi\left(\chi_{n k}\right), \pi\left(P_{n k}(t)\right)=\Phi\left(\chi_{n k}, t\right)$ and such that for $n=1,2, \cdots$ and all $t$ : 

(a') $\sum_{k=1}^{2^{n}} P_{n k}(t)=I$;
(b') $P_{n k}(t)=P_{n+1,2 k-1}(t)+P_{n+1,2 k}(t)$;
(c') $P_{n k}(t) P_{n j}(t)=0$ for $j \neq k$.

If $\mathcal{L}$ is the linear span of the functions $\left\{\chi_{n k}: 1 \leq k \leq 2^{n}, n \geq 1\right\}$ then properties $(a)-(c)$ and $\left(a^{\prime}\right)-\left(c^{\prime}\right)$ imply that

$$
\Psi\left(\sum_{k=1}^{2 n} a_{k} x_{n k}, t\right)=\sum_{k=1}^{2 n} a_{k} P_{n k}(t)
$$

is a well-defined map of $\mathfrak{L}$ into $\mathfrak{B}(\mathcal{H})$ for each $t$. Also (a)-(c) imply that $\mathfrak{Q}$ is a *-subalgebra of $C(X)$ which contains the constants; and properties (a), (c), and (d) imply that $\mathcal{Q}$ separates the points of $X$. Consequently, $\mathcal{Q}$ is

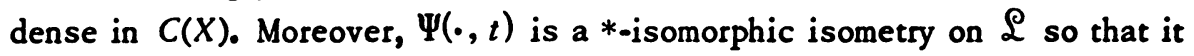
extends to a *-isomorphism of $C(X)$ into $B(H)$. Since $\Psi$ is continuous on $\mathcal{L} \times[0,1]$ its extension to $C(X) \times[0,1]$ is continuous. Because $\pi \Psi(f, t)=$ $\Phi(f, t)$ for $f$ in $\mathcal{L}$, the same equality holds for $f$ in $C(X)$. Finally, $\Psi\left(\chi_{n k}, 0\right)$ $=P_{n k}(0)=\psi\left(\chi_{n k}\right)$ and this implies that $\Psi(\cdot, 0)=\psi(\cdot)$. This completes the proof.

The proof of the next lemma is modeled on the proof of a theorem in [8] (see p. 268). Also, [1] contains related results.

7. Lemma. If $\{P(t)\}$ is a path of projections in $B(H)$ then there are paths of partial isometries $\{U(t)\}$ and $\{V(t)\}$ such that

$$
\begin{aligned}
U(t)^{*} U(t) & =P(0), & V(t)^{*} V(t) & =I-P(0), \\
U(t) U(t)^{*} & =P(t), & V(t) V(t)^{*} & =I-P(t), \\
U(0) & =P(0), & V(0) & =I-P(0) .
\end{aligned}
$$

Proof. Choose a $\delta>0$ such that $\|P(s)-P(t)\|<1$ whenever $|s-t|<$ ס. If $0 \leq t \leq \delta$ then $\|P(t)[P(0)-P(t)] P(t)\|<1$ and so

$$
A(t)=I+P(t)[P(0)-P(t)] P(t)
$$

is positive and invertible. In similar fashion

$$
B(t)=I+P(t)^{\perp}\left[P(0)^{\perp}-P(t)^{\perp}\right] P(t)^{\perp}
$$

is positive and invertible. Hence $\left\{A(t)^{-1 / 2}\right\}$ and $\left\{B(t)^{-1 / 2}\right\}$ are continuous paths for $0 \leq t \leq \delta$. Therefore $U(t)=P(t) A(t)^{-1 / 2} P(0)$ and $V(t)=P(t)^{\perp} B(t)^{-1 / 2} P(0)^{1}$ are continuous on $[0, \delta]$. By the arguments used in [8], $U(t)$ and $V(t)$ are partial isometries with the desired initial and final spaces. Also $U(0)=P(0)$ and $V(0)=P(0)^{\perp}$. 
Similarly there are paths $\{\tilde{U}(t)\}$ and $\{\tilde{V}(t)\}$ of partial isometries with $\tilde{U}(\delta)=P(\delta), \widetilde{V}(\delta)=P(\delta)^{\perp}, \tilde{U}(t)^{*} \widetilde{U}(t)=P(\delta), \tilde{U}(t) \tilde{U}(t)^{*}=P(t), \widetilde{V}(t)^{*} \tilde{V}(t)=P(\delta)^{\perp}$, $\tilde{V}(t) \tilde{V}(t)^{*}=P(t)^{\perp}$ for $\delta \leq t \leq 2 \delta$. If $U(t)=U(\delta) \tilde{U}(t)$ and $V(t)=V(\delta) \tilde{V}(t)$ for $\delta \leq t \leq 2 \delta$ then this results in continuous paths on $[0,2 \delta]$ which have the desired properties. The proof is completed by induction.

8. Theorem. If $X_{1}$ and $X_{2}$ have the $C^{*}$-covering homotopy property then so does their disjoint union.

Proof. If $X$ is the disjoint union of $X_{1}$ and $X_{2}$, let $\Phi: C(X) \times[0,1]$ $\rightarrow \mathfrak{B}(\mathcal{H}) / \mathcal{K}$ be a continuous map such that $\Phi(\cdot, t)$ is a $*$-isomorphism for each $t$ and such that there is a $*$-isomorphism $\psi: C(X) \rightarrow \mathscr{B}(\mathcal{H})$ with $\pi \psi(\cdot)=\Phi(\cdot, 0)$. If $\chi_{1}$ and $\chi_{2}$ are the characteristic functions of $X_{1}$ and $X_{2}$, respectively, then according to Lemma 5 there is a path $\left\{P_{1}(t)\right\}$ of projections such that if $P_{2}(t)=P_{1}(t), \pi\left(P_{i}(t)\right)=\Phi\left(\chi_{i}, t\right), P_{i}(0)=\psi\left(\chi_{i}\right)$ for $i=1,2$. For $i=1,2$ put $P_{i}=P_{i}(0), \mathcal{H}_{i}=P_{i}(\mathcal{H}), \mathcal{K}_{i}=$ the compact operators on $\mathcal{H}_{i \bullet}$ Then $\mathfrak{B}_{\left(\mathcal{H}_{i}\right)}$ and $\mathfrak{B}^{i}\left(\mathcal{H}_{i}\right) / K_{i}$ can be identified with $P_{i} \mathfrak{B}(\mathcal{H}) P_{i}$ and $\left.\pi\left(P_{i}\right)[\mathcal{B O H}) / K\right]_{\pi}\left(P_{i}\right)=G_{i}$, respectively. The preceding lemma implies that there are paths of partial isometries $\left\{U_{i}(t)\right\}, i=1$ and 2 , such that $U_{i}(0)=P_{i}, U_{i}(t)^{*} U_{i}(t)=P_{i}, U_{i}(t) U_{i}(t)^{*}$ $=P_{i}(t)$. Define $\Phi_{i}: C\left(X_{i}\right) \times[0,1] \rightarrow \mathbb{Q}_{i}$ and $\psi_{i}: C\left(X_{i}\right) \rightarrow P_{i} B(\mathcal{H}) P_{i}$ by

$$
\begin{gathered}
\Phi_{i}(f, t)=\left(U_{i}(t)\right)^{*} \Phi(f, t) \pi\left(U_{i}(t)\right), \\
\psi_{i}(f)=P_{i} \psi(f) P_{i}=\psi(f)
\end{gathered}
$$

for each $f$ in $C\left(X_{i}\right)_{0}\left(C\left(X_{i}\right)\right.$ is identified with the obvious ideal of $\left.C(X)_{0}\right)$

By hypothesis there is a continuous map $\left.\Psi_{i}: C\left(X_{i}\right) \times[0,1] \rightarrow P_{i} \mathfrak{B O H}\right) P_{i}$ such that $\Psi_{i}(\cdot, 0)=\Psi_{i}(\cdot), \pi \Psi_{i}=\Phi_{i}$, and $\Psi_{i}(\cdot ; t)$ is a *-isomorphism for each $t$ (with $\Psi_{i}(1, t)=P_{i}$ ). Define $\Psi: C(X) \times[0,1] \rightarrow B(K)$ by

$$
\Psi(f, t)=U_{1}(t) \Psi_{1}\left(f x_{1}, t\right) U_{1}(t)^{*}+U_{2}(t) \Psi_{2}\left(f x_{2}, t\right) U_{2}(t)^{*}
$$

for $0 \leq t \leq 1$ and $f$ in $C(X)$. It is left to the reader to show that $\pi \Psi=\Phi$, $\Psi(\cdot, 0)=\bar{\Psi}(\cdot)$, and $\Psi(\cdot, t)$ is a *-isomorphism for each $t$.

\section{REFERENCES}

1. H. Araki, M.-S. B. Smith and L. Smith, On the homotopical significance of the type of von Neumann algebra factors, Comm. Math. Phys. 22 (1971), 71-88. MR 44 \#5783.

2. L. G. Brown, Operator algebras and algebraic K-theory (to appear).

3. L. G. Brown, R. G. Douglas and P. A. Fillmore, Extensions of $C^{*}$-algebras, operators with compact self-commutators, and K-homology, Bull. Amer. Math. Soc. 79 (1973), 973-978.

4. - Unitary equivalence modulo the compact opertors and extensions of $C^{*}$ algebras, Proc. Conf. on Operator Theory, Lecture Notes in Math., vol. 345, Springer-Verlag, New York, 1973, pp. 58-128. 
5. P. R. Halmos, A Hilbert space problem book, Van Nostrand, Princeton, N. J., 1967. MR 34 \#8178.

6. J. Kaminker and C. Schochet, Ext $(X)$ from a homological point of view, Proc. Conf. on Operator Theory, Lecture Notes in Math., vol. 345, Springer-Verlag, New York, 1973, pp. 129-140.

7. E. Michael, Continuous selections. I, Ann. of Math. (2) 63 (1956), 361-382. MR 17, 990.

8. F. Riesz and B. Sz. Nagy, Leçons $d$ 'analyse fonctionelle, 2nd ed., Akad. Kiadó, Budapest, 1953; English transl., Functional analysis, Ungar, New York, 1955. MR 15, 132; 17, 175.

DEPARTMENT OF MATHEMATICS, INDIANA UNIVERSITY, BLOOMINGTON, INDIANA 47401 